Journal for

ImmunoTherapy of Cancer

\title{
Point mutation in CD19 facilitates immune escape of B cell lymphoma from CAR-T cell therapy
}

\author{
Zhen Zhang, ${ }^{1}$ Xinfeng Chen, ${ }^{1}$ Yonggui Tian, ${ }^{1}$ Feng Li (D) , ${ }^{1}$ Xuan Zhao, ${ }^{1}$ Jinyan Liu, ${ }^{1}$ \\ Chang Yao, ${ }^{1}$ Yi Zhang (1) ${ }^{1,2,3,4}$
}

To cite: Zhang Z, Chen $X$, Tian Y, et al. Point mutation in $C D 19$ facilitates immune escape of $B$ cell lymphoma from CAR-T cell therapy. Journal for ImmunoTherapy of Cancer 2020;8:e001150. doi:10.1136/ jitc-2020-001150

- Additional material is published online only. To view, please visit the journal online (http://dx.doi.org/10.1136/jitc2020-001150).

$\mathrm{ZZ}, \mathrm{XC}$ and $\mathrm{YT}$ contributed equally.

Accepted 13 September 2020

Check for updates

(C) Author(s) (or their employer(s)) 2020. Re-use permitted under CC BY-NC. No commercial re-use. See rights and permissions. Published by BMJ.

${ }^{1}$ Biotherapy Center, the First Affiliated Hospital of Zhengzhou University, Zhengzhou, Henan, China

${ }^{2}$ School of Life Sciences, Zhengzhou University, Zhengzhou, Henan, China

${ }^{3}$ Cancer Center, the First Affiliated Hospital of Zhengzhou University, Zhengzhou, Henan,

China

${ }^{4}$ Henan Key Laboratory for Tumor Immunology and Biotherapy, Zhengzhou, Henan, China

Correspondence to

Professor Yi Zhang;

yizhang@zzu.edu.cn

\section{ABSTRACT}

Background Tumor relapse due to mutation in CD19 can hinder the efficacy of chimeric antigen receptor (CAR)-T cell therapy. Herein, we focused on lymphoma patients whose $B$ cells exhibited a point mutation in CD19 of B cells after CAR-T cell infusion.

Methods The CAR-T and CD19 ${ }^{+} B$ cells from peripheral blood or bone marrow were assessed using flow cytometry. Genome sequencing was conducted to identify the molecular characteristics of CAR-T and CD19 ${ }^{+} B$ cells from pre-relapse and postrelapse samples. CD19 in CARs comprising single chain fragments variable (scFV) antibody with FMC63 or 21D4 was constructed. The cytotoxic efficacy of CAR-T cells was also evaluated via in vitro and in vivo experiments.

Results A patient with high-grade B cell lymphoma exhibited complete response, but the lymphoma relapsed in her left breast at 6 months after CD19 CAR (FMC63)-T cell infusion. A mutation was found in exon 3 of $C D 19$ (p.163. R-L) in malignant B cells of the patient. In two lymphoma patients who exhibited resistance to CAR-T cell therapy, a mutation was detected in exon 3 of $C D 19$ (p.174. L-V). Functional analysis revealed that FMC63 CAR-T cells exhibited antitumor ability against wild-type $\mathrm{CD} 19^{+}$cells but were unable to eradicate these two types of mutated CD19+ cells. Interestingly, 21D4 CAR-T cells were potentially capable of eradicating these mutated $\mathrm{CD} 19^{+}$cells and exhibiting high antitumor capacity against CD19 ${ }^{+}$cells with loss of exon 1,2 , or 3 .

Conclusions These findings suggest that point mutation can facilitate immune escape from CAR-T cell therapy and that alternative CAR-T cells can effectively eradicate the mutated B cells, providing an individualized therapeutic approach for lymphoma patients showing relapse.

\section{BACKGROUND}

Cancer immunotherapy based on chimeric antigen receptor (CAR)-T cells against CD19 has been used successfully to treat B-cell malignancies. ${ }^{1} 2$ Unfortunately, a significant number of responding patients have been reported to eventually exhibit relapse. ${ }^{3}$ Recently, there are two major types of relapse were reported, including antigen loss (CD19and positive $\left(\mathrm{CD} 19^{+}\right)$, following analysis of CD19 expression in B cells by using clinical flow cytometry. An estimated $20 \%-30 \%$ of relapses after CD19 CAR-T cell therapy are attributed to the antigen loss, indicating an urgent need for investigating the mechanisms underlying recurrence and for improving the efficacy of CAR-T cell therapy. ${ }^{45}$ Interestingly, one of the specific mechanisms of tumor escape that has been reported suggests that exon mutations affecting the CD19 gene and its splicing isoforms, leading to the disappearance of CD19 epitope that is recognized by the FMC63-based antigenbinding moiety of CD19 CAR. ${ }^{6}$ Other mechanisms, such as induction of a myeloid switch in the B-cell acute lymphoblastic leukemia (ALL) patients with rearrangements of the mixed lineage leukemia (MLL) gene or the persistence of minor CD19- clones have also been reported. ${ }^{78}$ Dual CARs targeting two different antigens, namely CD19 and CD123, represent an effective strategy to prevent antigen-loss associated relapses. ${ }^{4}$ In the $\mathrm{CD} 19^{+}$ relapsed patients, CD19 remains present on the B-ALL cell surface, while the CAR-T cells can become exhausted in vivo. ${ }^{9}{ }^{10}$ Moreover, these patients still have a poor prognosis with re-infusion of autologous CAR-T cells. ${ }^{11} \mathrm{~A}$ rare case has been reported in which the CAR gene was transduced into a single leukemic B cell during CTL019 (tisagenlecleucel, Kymriah, Novartis) generation and the CD19 CAR expression directly mediated the loss of CD19 in leukemia cells, thereby hindering its detection by standardized flow cytometry. CD19 transcripts and specific proteins are preserved and resistant to CD22 CAR-T cell therapy. ${ }^{12}$ Furthermore, there are few reports on $\mathrm{CD} 19^{+}$relapse in lymphoma patients, and the strategy to overcome this issue is still not well defined.

In this study, we have reported a 24-year-old female patient with high-grade $B$ cell lymphoma (HGBCL) who achieved complete response (CR) after being treated with autologous CD19 CAR (FMC63)-T cells. 
Unfortunately, $\mathrm{CD} 19^{+}$relapse occurred in her left breast after 6 months, which was associated with a point mutation in exon 3 of CD19. Despite the continuous generation of CAR-T cells possessing antitumor function against wild type $\mathrm{CD} 19^{+} \mathrm{B}$ cells, they could not eradicate the mutated $\mathrm{CD} 19^{+}$cells in vivo. Interestingly, another CD19 CAR, namely 21D4, transduced T-cells exhibited a more enhanced antitumor capacity to eradicate the mutated $\mathrm{CD} 19^{+}$cells or exon 1-deleted, 2-deleted, or 3-deleted H322-CD19 cells than that of FMC63 CAR-T cells. Moreover, we found no mutation in $\mathrm{CD} 19^{+}$tumor cells from bone marrow of one lymphoma patient who exhibited long-term remission after CAR-T cell therapy. Conversely, a point mutation was detected in $\mathrm{CD} 19^{+}$cells from samples of lymphoma samples who had no response (NR) to autologous FMC63 CAR-T cells, but these mutated B cells could be killed by 21D4 CAR-T cells, indicating the potential of 21D4 CAR-T cells in treatment of patients with CD19 mutations.

\section{METHODS}

\section{Study design}

The clinical trial in which patients with B cell malignancies participated was conducted at the First Affiliated Hospital of Zhengzhou University and has been reported previously (ClinicalTrials.gov number, NCT03156101). ${ }^{13}$ End points for this study included safety, overall survival, duration of response, and CD19 CAR-T cellular kinetics. These patients were subjected to lymphodepleting chemotherapy with fludarabine $\left(25 \mathrm{mg} / \mathrm{m}^{2}\right.$ body surface area per day) for 3 days, and cyclophosphamide $\left(500 \mathrm{mg} / \mathrm{m}^{2}\right.$ body surface area per day) for 2 days, followed by infusion of CD19 CAR-T cells after 3-5 days. Peripheral blood and bone marrow samples were drawn to evaluate the CAR-T and $\mathrm{CD} 19^{+} \mathrm{B}$ cells kinetics. Sequencing of RNA (RNAseq) and DNA (DNA-seq) was performed to evaluate the CAR-T function and mutation of B cells. Positron emission tomography (PET)-CT or CT was performed to evaluate the overall disease burden. Production of CAR-expressing $\mathrm{T}$ cells were produced for the experiments as previously described. ${ }^{13}$ Patients and healthy donors provided written informed consents according to the Declaration of Helsinki presented at the International Conference on Harmonization Guidelines for Good Clinical Practice. All relevant ethical regulations were followed in this study.

\section{Lentiviral constructs and CAR-T cell generation}

CD19 CARs comprising a single chain fragment variable (scFv) derived from clone FMC63 or 21D4, 4-1BB (CD137) costimulatory, and CD3-zeta domains were generated as previously described. ${ }^{13}$ Peripheral blood samples drawn from patients or healthy donors were used to produce CD19 CAR-T cells. The transduced T-cells were cultured for about 10 days and the transduction efficiency was evaluated by flow cytometry before conducting infusion.

To generate CD19 or mutated CD19 (p.163. R-L) expressing vectors, the complete gene as well as the
CD19 exon 1, 2, 3, or 4 deleted versions were synthesized commercially (Sheng gong, Shanghai) and cloned into pCDH-EF1-IRES-Puro vector. Lentiviral particles were generated by transfection of the above-mentioned constructs into $293 \mathrm{~T}$ cells using Lipofectamine-2000 (Invitrogen). Viral supernatants were harvested 48 hours post-transfection and used to infect luciferase expressing H322 or A549 cell lines in the presence of polybrene (4 $\mu \mathrm{g} / \mathrm{mL})$.

\section{Cell lines}

A549, H322, 293T, and Raji cell lines were obtained from the Cell Bank of the Chinese Academy of Sciences. Cell lines were cultured in DMEM (Gibco) supplemented with $10 \%$ fetal bovine serum (FBS; Hyclone) and antibiotics (Penicillin and Streptomycin; Gibco) at $37^{\circ} \mathrm{C}$ with a continuous supply of $5 \% \mathrm{CO}_{2}$. These cells were then infected with pLenti-CMV-luc2-IRES-Puro virus to express the firefly luciferase.

\section{Flow cytometry}

Peripheral blood mononuclear cells (PBMCs) were isolated from the peripheral blood samples drawn from patients. Briefly, $1 \times 10^{6}$ cells were washed with phosphatebuffered saline (PBS) and resuspended in fluorescence activated cell sorting (FACS) buffer consisting of PBS and 2\% FBS. Cells were then incubated with anti-CD3PE-cy7 (clone UCHT1; BD Pharmingen), CD19 (20-291) protein-FITC (ACRO Biosystems), or anti-CD19-PE (clone HIB19; BioLegend) antibodies for $30 \mathrm{~min}$. Flow cytometry analysis was performed on BD FACS CantoII (BD, USA).

\section{Cytotoxicity assays}

The ability of CD19 CAR-T cells (FMC63 or 21D4) to kill the target cells expressing CD19 was evaluated by flow cytometry or bioluminescent imaging (BLI) using the IVIS Spectrum Imaging System (PerkinElemer). Approximately $3 \times 10^{4} \mathrm{CD} 19^{+}$cells from bone marrow preinfusion and postinfusion of CAR-T cells, CD19-H322 cells, CD19-A549 cells, mutated CD19 (p.163. R-L)-H322 cells, mutated CD19 (p.163. R-L)-A549 cells, and exon 1-deleted, 2-deleted, 3-deleted, or 4-deleted H322-CD19 cells were incubated with CAR-T cells for 6 or 24 hours at various effector:target (E:T) ratios. The lysis of target cells was determined by Annexin-V-PI staining. The BLI was performed and the viabilities of target cells were determined based on their photon intensities. Tumor cells alone were used as the control.

\section{Quantitative real-time PCR}

Genomic DNA was isolated from PBMCs that were collected before the treatment and at multiple timepoints after treatment by using the DNeasy blood \& tissue kit (Qiagen). The kinetics of CAR-modified cells in vivo was assessed using validated PCR primers specific to the $4-1 \mathrm{BB}$ and $\mathrm{CD} 3 \zeta$ fusion gene as previously described. ${ }^{1415}$ 


\section{RNA sequencing}

CAR-T cells generated from PBMCs before infusion and at day 14 and 180 post-treatment were collected by using the cell sorter Moflo-XDP (Beckman-Coulter). CD19 ${ }^{+}$ cells from bone marrow or tumor tissues before treatment and at days 7 and 180 post-treatment were collected to evaluate the molecular characteristics of B cells. Total RNA was isolated using TRIzol reagent (Invitrogen) and treated with RNase-free DNaseI at $37^{\circ} \mathrm{C}$ for $20 \mathrm{~min}$. Integrity of RNA was assessed on the Agilent TapeStation (RIN), followed by preparation for sequencing using the TruSeq RNA v2 prep (Illumina) as previously described. ${ }^{12}$ RNA-sequencing and data analysis were performed at BGI (Wuhan, China).

\section{Whole-exome sequencing}

Genomic DNA was extracted from the bone marrow or tumor tissue according to manufacturer's instructions (Promega). ${ }^{16} 17$ The enriched DNA fragments were sheared to $150-200 \mathrm{bp}$ fragments on an average and were subjected to standard Illumina Genome Analyzer library preparation according to manufacturer's protocol. High-quality reads were aligned to the reference human genome (build hg19) using the Burrows-Wheeler Aligner (BWA-v0.7.15). All variants were subjected to visual inspection. Sequencing and data analysis were performed at BGI (Wuhan, China).

\section{In vivo animal models}

We established Raji/Luc lymphoma model via intravenous injection through tail, and CD19-H322/Luc and mutated CD19-H322/Luc solid tumor models via subcutaneous inoculation using 6-8 weeks old female SCID-beige mice. The volume of these cells $\left(1 \times 10^{6}\right)$ per injection was 100 $\mu \mathrm{L}$. Thereafter, $1 \times 10^{7}$ CAR-T cells were resuspended in PBS and injected through the tail vein. Then, the tumor burdens were evaluated by BLI.

\section{Statistical analyses}

All the data were analyzed using Prism V.7.0 (GraphPad Software) and SPSS V.17.0 software. Paired or unpaired two-tailed t-tests were performed to analyze the cytotoxicity of CAR-T cells. Survival curves were analyzed using the log-rank test. Statistical significance was defined as $\mathrm{p}<0.05$.

\section{RESULTS}

\section{Clinical characteristics of the $\mathrm{CD}^{+} 9^{+}$relapsed patient}

Patient 1 who was diagnosed with HGBCL showed progression after chemotherapy with R-CHOP, and Hyper CVAD $\mathrm{A}$ and $\mathrm{B}$ regimen. However, she was found to be refractory to other regimens, including MA (methotrexate and cytarabine), alternating IVAC (etoposide, ifosfamide, cytarabine), and combined treatment with intrathecal injection two times per cycle (methotrexate, cytarabine, and dexamethasone). Approximately $77.6 \%$ and $88.4 \%$ clonal mature B lymphocytes were found to persist in her bone marrow and cerebrospinal fluid, respectively. Thereafter, she was enrolled in a clinical trial of BinD19, which included treatment with a CD19-directed CAR (FMC63)-T cell product. Following lymphodepletion, she was infused (splitting with dose escalation) with $2.23 \times 10^{8}$ total $\mathrm{T}$ cells, comprising $2.18 \times 10^{7}$ CAR-T cells for over 3 days. The major therapeutic schedule followed is shown in figure 1A. She had fever on day 3 after infusion of CAR-T cells, as tocilizumab (160 mg), methylprednisolone $(40 \mathrm{mg})$, and dexamethasone $(10 \mathrm{mg})$ were administered repeatedly to control cytokine release syndrome. PET-CT scan images revealed multiple lymphadenopathy and elevated standard uptake values in the breast, uterus, bilateral ovary, liver, bones, and subcutaneous mass before treatment (figure 1B). After infusion of CAR-T cells, she achieved CR by day 60 . However, the CD $19^{+}$ relapse occurred in her left breast on day 180 . She then received two times the amount of anti-programmed cell death protein 1 therapy and eventually the augmented tumor mass was removed by surgical resection. This patient recovered and exhibited CR state by April 2020 (figure 1A,B).

\section{Kinetics of CAR-T and B cells in vivo}

We assessed $\mathrm{CD}^{+}{ }^{+}$CAR-T cells by flow cytometry in blood sample of patient obtained after infusion. The proliferative burst of CAR-T cells was apparent on day 14 $(10.8 \%)$ and these cells still persisted until day 180 (3.1 $\%$ ), which is the period of relapse (figure 1C). After PD-1 blocking therapy and surgery, this patient achieved CR again and the percentage of CAR-T cells was $1.4 \%$ and $1.1 \%$ in peripheral blood and bone marrow, respectively (day 579, figure 1C). Meanwhile, the expansion and long persistence of CAR-T cells were confirmed via quantitative real-time (qRT)-PCR using CAR-specific primers (figure 1D). We also observed a directed relationship between in vivo CAR-T and abnormal $\mathrm{B}$ cells in peripheral blood. Our data indicated that the $\mathrm{CD} 19^{+} \mathrm{B}$ cells were significantly decreased on day $14(7.7 \%)$ and disappeared on day 28 (figure 1C). Additionally, the proportion of malignant $\mathrm{CD} 19^{+} \mathrm{B}$ cells in bone marrow was $61.7 \%$ at the baseline that rose up to $95.4 \%$ on day 7 . However, it decreased to $0.3 \%$ on day 17 after CAR-T cell infusion (data not shown). At day 180, the patient experienced relapse, as noted by abundant infiltration $(84.1 \%)$ of $\mathrm{CD} 19^{+} \mathrm{B}$ cells in the left breast tissues. However, the expression of CD19 was very low in blood $(1.0 \%)$ and bone marrow $(1.2 \%)$ (figure $1 \mathrm{E}$ ). These results suggest that tumor cells could evade CAR-T cell-mediated recognition and clearance, despite the persistence exhibited by CAR-T cells.

\section{Characteristics of CAR-T and B cells derived from patient postrelapse}

To further characterize the CAR-T and B cells derived from the patient at different points, RNA-seq was performed (online supplemental table S1,2). Our results showed that the inhibitor markers, such as LAG3, TIGIT, and CTLA-4 


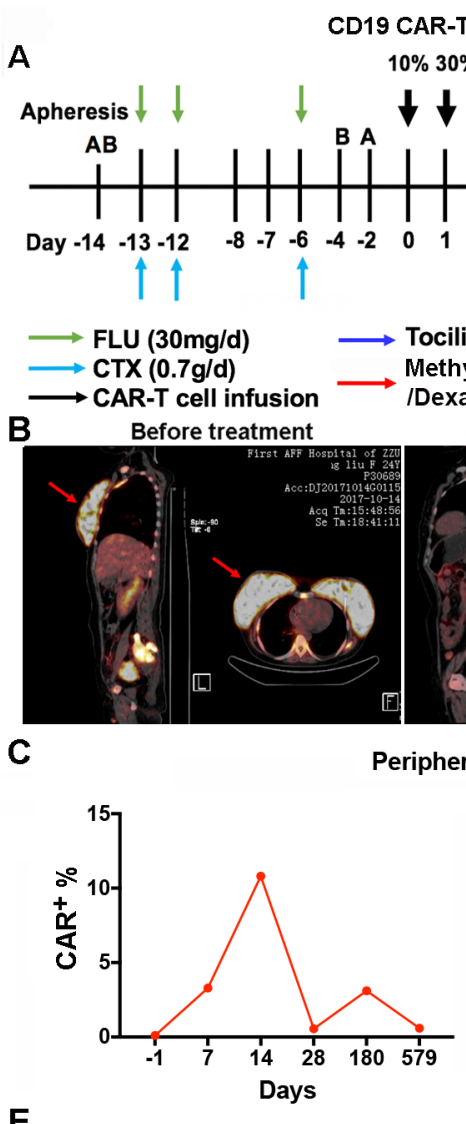

19 CAR-T cell infusion

\section{A} 30\% 60\% Dex (10/15mg)

Follow up

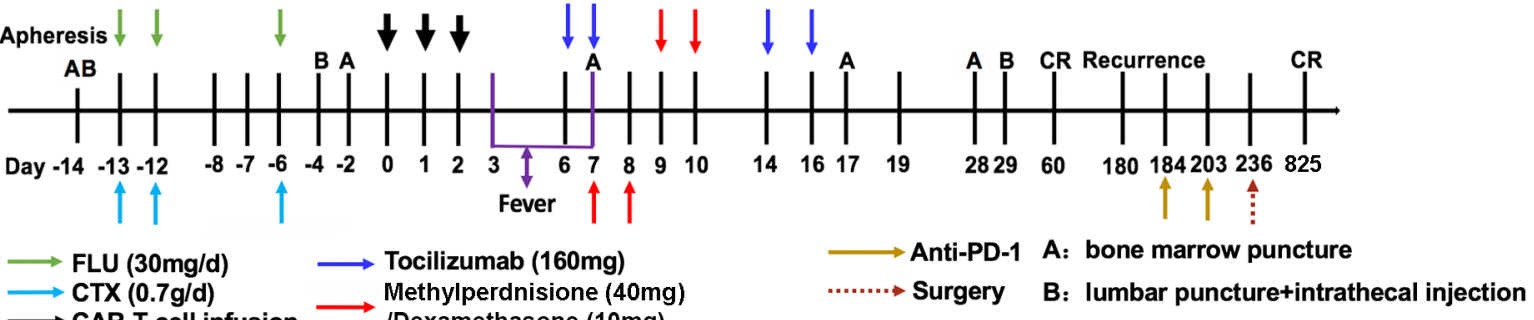

B

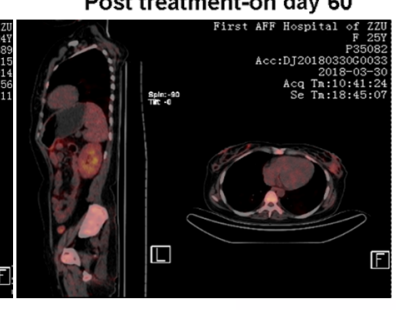

Peripheral Blood

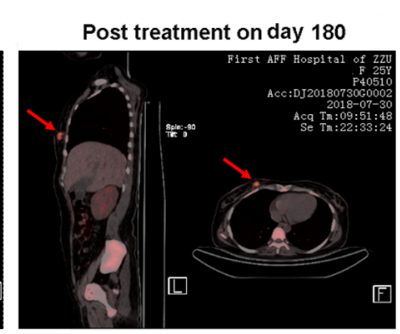

D
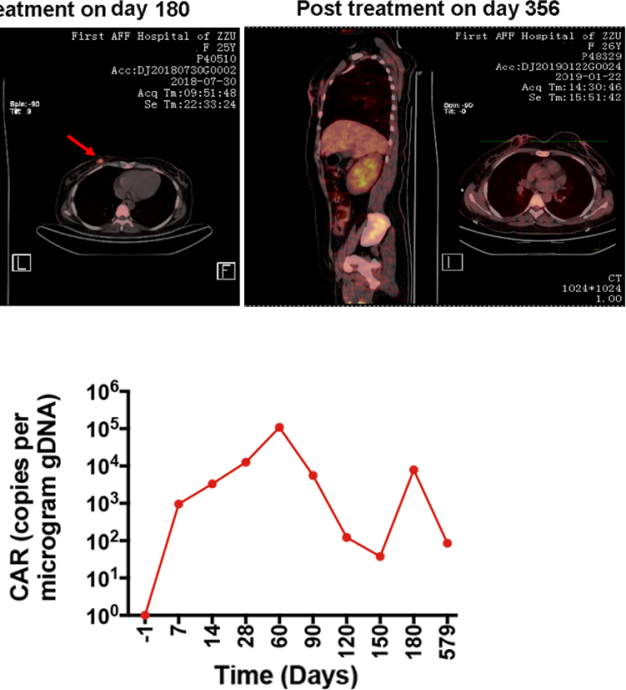

E

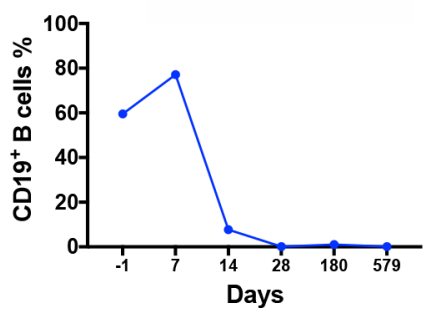

Day 180
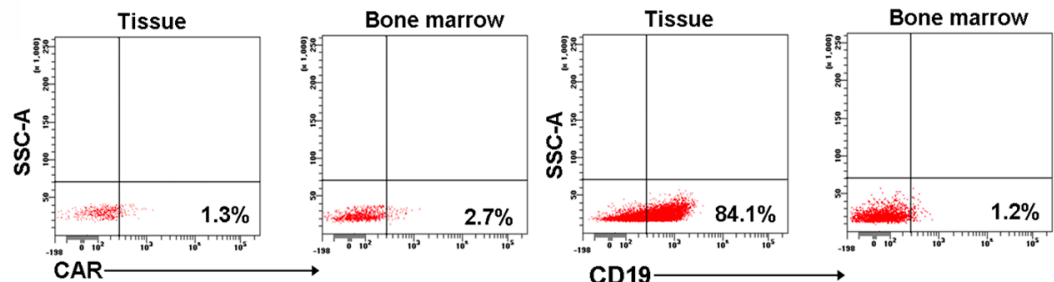

Figure 1 Treatment regimen and kinetics of CD19 CAR-T cells in a patient with high-grade B cell lymphoma. (A) Timeline of the CAR-T protocol conducted for one patient with lymphoma. (B) The positron emission tomography-CT scan images of this patient before treatment, and on days 60, 180, and 356 post-CD19 CAR-T cell infusion are shown. (C) The percentage of CD19 cells derived from peripheral blood mononuclear cells was detected by flow cytometry analysis. (D) Time course analysis of expression levels of CD19 CAR transgene by quantitative real-time-PCR. (E) The fractions of CAR-T and CD19 $B$ cells in the tissue and bone marrow were detected by flow cytometry on day 180. CAR, chimeric antigen receptor; CR, complete response; CTX, cyclophosphamide; FLU, fludarabine; PD1, programmed cell death protein 1; SSC-A,side scatter-A.

exhibited a lower expression level, but the expression of PDCD1 was higher in CAR-T cells at the time of relapse than that of CAR-T cells in pre group (figure 2A). The transcription factors TBX21, ZEB2, and ID2 are critical for T-cell exhaustion in cancer, ${ }^{18}$ but their expression had no obvious difference in CAR-T cells on days 14 and 180. Meanwhile, the exhausted marker NR4A1 was highly expressed in CAR-T cells on day 180 compared with that in CAR-T cells before infusion and on day 14, while the expression level of memory differentiation related genes such as EOMES and TCF7 was high in CAR-T cells on day 180 , as well as the memory stem cell associated genes such as CCR7, FAS (encoding CD95), PTPRC (encoding CD45RA), and SELL (encoding CD62L, figure 2A). Thus, these genes may cause a difference in persistence of
CAR-T cells in vivo. Herein, CAR-T cells at the relapse also expressed high level of GZMA, IFN- $\gamma, G Z M B$, and $R P F 1$, suggesting that these cells might have possessed potential antitumor ability in vivo. To understand the reason of relapse in this patient, we further analyzed the gene profile and mutations in $\mathrm{CD} 19^{+} \mathrm{B}$ cells derived from bone marrow and tumor tissues. Among the genes whose expression was changed in B cells, some transcription factors captured our attention. For example, the IRF4, MEF2B, BCL6, and TCF3, which are associated with progression of lymphoma, were significantly upregulated on day 180, as well as the $\mathrm{B}$ cell receptor $(C D 79 B)$, Toll like receptor (MYD88), and the NF-kappa B protein (RELA). PRDM1, a master regulator of plasma-cell differentiation, was downregulated in relapsed tumors and its loss may 

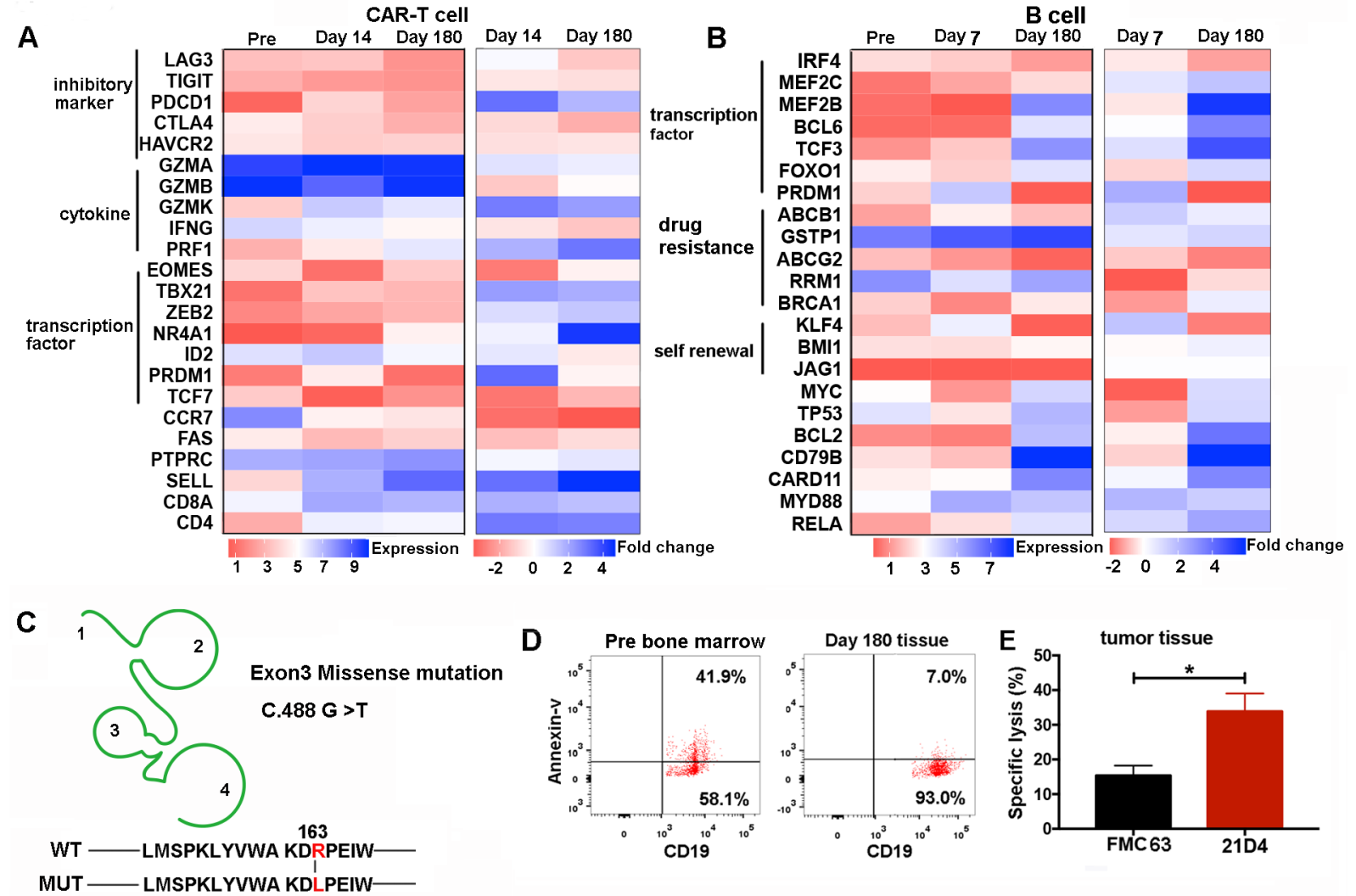

Figure 2 The molecular characteristics of CAR-T and CD19+ B cells. (A-B) Expression levels of the selected genes in CAR-T and $\mathrm{CD} 19^{+} \mathrm{B}$ cells before and after treatment were detected by RNA-seq. Left panel: the gene expression details of CAR-T or CD19 $\mathrm{B}$ cells in pretreatment and post-treatment samples; right panel: the fold change in the expression of selected genes in post-treatment samples derived CAR-T or CD19 ${ }^{+} \mathrm{B}$ cells (relative to the pretreatment value). (C) The CD19 ${ }^{+} \mathrm{B}$ cells isolated from tumor tissues of relapsed patient exhibited a missense mutation in exon 3 (P. 163 R>L/C.488 G>T). (D) The autologous CAR (FMC63)- $\mathrm{T}$ cells cocultured with $\mathrm{CD} 19^{+} \mathrm{B}$ cells were derived from bone marrow before treatment and from tumor tissues after relapse at a ratio of 10:1. The CAR-T cells are potentiated to eradicate normal CD19 ${ }^{+}$cells, but not the mutated target cells. (E) The FMC63 and 21D4 CAR-T cells were incubated with target cells derived from tumor tissues. ${ }^{*} p<0.05$. CAR, chimeric antigen receptor.

contributed to the poor prognosis of patients. ${ }^{19}$ Meanwhile, no difference was observed in the expression level of stem cell-related and drug resistance-related genes in bone marrow before and after CAR-T cell therapy (on day 7 and 180, figure 2B).

For further analysis of whether the malignant B cells had a mutation or not, we performed whole-exome sequencing (WES). Interestingly, the p.163 (exon3) $\mathrm{R}>\mathrm{L}$ site mutation was identified in $\mathrm{CD} 19^{+}$cells $(95 \%)$ that were isolated from relapsed tumor tissues (figure 2C). However, the $\mathrm{CD} 19^{+}$cells derived from bone marrow before therapy had no mutation (data not shown). Further functional analysis of CAR-T cells revealed that specific lysis of $\mathrm{CD} 19^{+}$cells derived from bone marrow before treatment was higher than those derived from relapsed tissue, indicating that the relapsed $\mathrm{CD} 19^{+} \mathrm{B}$ cells could not be eradicated by autologous CAR-T cells (figure 2D). We then constructed CD19 CARs comprising scFV antibody fragments, FMC63 and 21D4, and obtained two types of CD19 CAR-T cells. When compared with FMC63, the 21D4 CAR-T cells exhibited better effectorspecific function against mutated $\mathrm{CD} 19^{+} \mathrm{B}$ cells derived from tumor tissues (figure 2E). In summary, point mutation of CD19 confers resistance to autologous FMC63 CAR-T cells, and sensitivity to 21D4 CAR-T cells.

\section{Cytotoxic effect of FMC63 and 21D4 CAR-T cells}

To validate the antigen-specific cytotoxic effect of 21D4 CAR-T cells, we conducted an in vitro experiment. Wild type or mutated (exon3, p.163. R-L) CD19 ${ }^{+} \mathrm{H} 322$ and A549 cells expressing luciferase were established and the expression of CD19 can be detected at the similar level in these tumor cells through labeling with anti-CD19 antibody (HIB19, online supplemental figure S1A, B). CAR-T cells were then incubated with the target cells for 24 hours, and H322 and A549 cells as a negative control. Our results showed that both the FMC63 and 21D4 CAR-T cells exhibited a low antitumor activity against H322 and A549 cells (online supplemental figure S2A-C). As shown in figure 3A, FMC63 CAR-T cells demonstrated potent cytotoxicity against $\mathrm{CD} 19^{+}$tumor cells, but not against CD19 mutated cells $(10: 1$, H322-mCD19, 2.90E+07 $\pm 9.68 \mathrm{E}+05$; A549-mCD19， 2.26E+07 $\pm 3.23 \mathrm{E}+06)$. However, 21D4 CAR-T cells exhibited similar cytotoxicity against both 
A

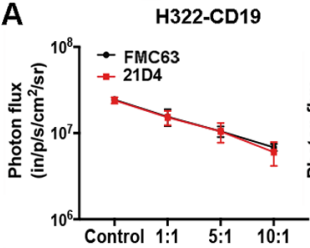

B

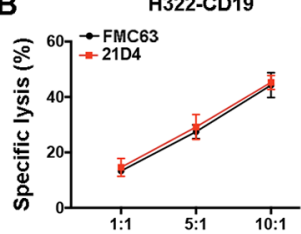

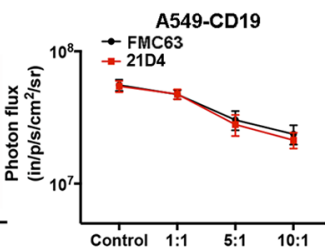
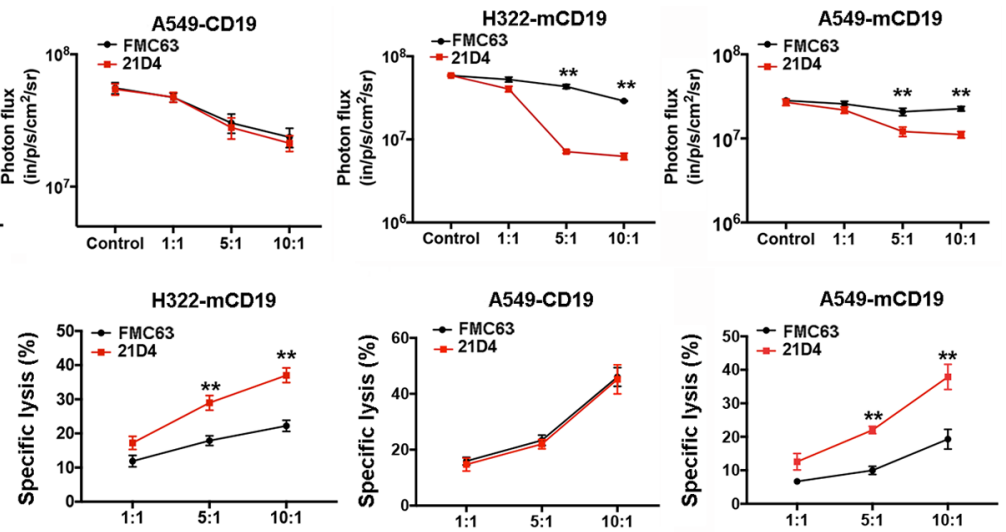

C
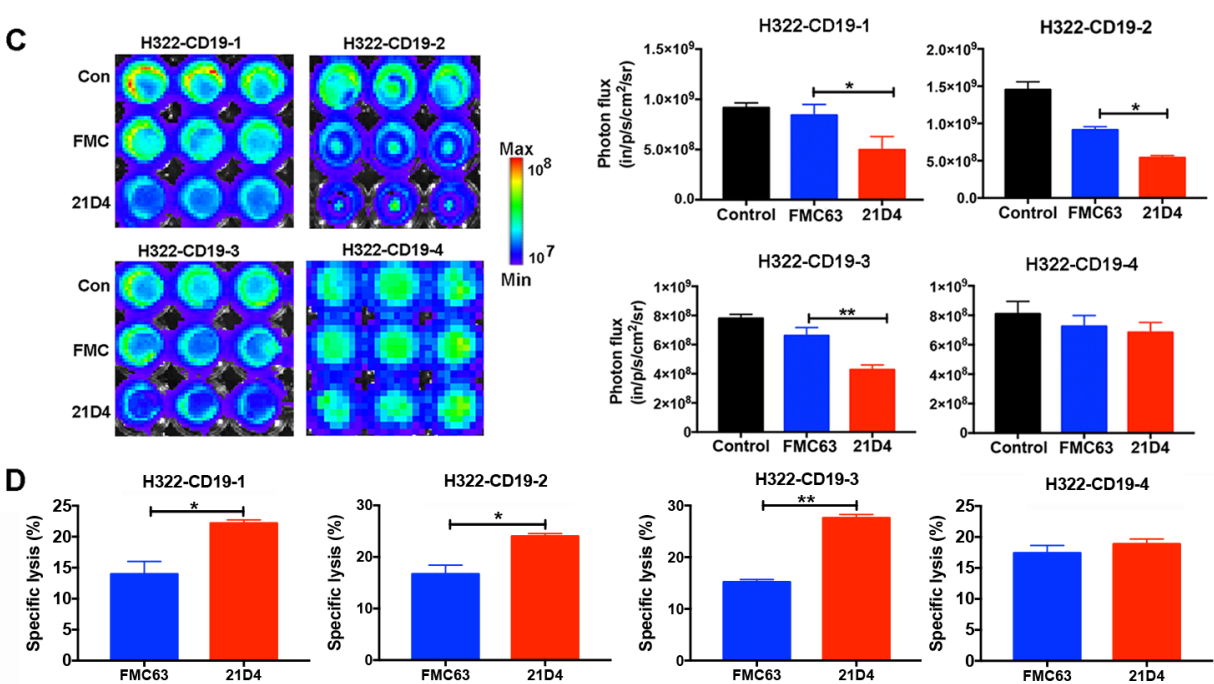

Figure 3 21D4 CAR-T cells show enhanced effector-specific function following stimulation by loss of exon or mutated CD19 $9^{+}$targeted cells in vitro. (A) FMC63 and 21D4 CAR-T cells were incubated with wild type or mutant (exon3, p.163). (R-L) CD19-H322 and A549 cells for 24 hours. Then the viabilities of target cells were evaluated based on bioluminescent imaging intensities. (B). Tumor cells were incubated with CAR-T cells for 6 hours. Then the tumor cells were collected and stained with Annexin-V/PI to determine specific lysis. (C) The CD19+ cells with deleted exon 1, 2, 3, or 4 were collected and co-cultured with CAR-T cells for 24 hours. Then the lysis of target cells was evaluated based on BLI intensities. (D) The specific lysis of CD19 ${ }^{+}$ tumor cells with deleted exon was detected by flow cytometry. ${ }^{*} p<0.05,{ }^{* *} p<0.01$. CAR, chimeric antigen receptor.

wild type and CD19 mutated cells (10:1, H322-mCD19, $6.25 \mathrm{E}+06 \pm 1.27 \mathrm{E}+06 ; \mathrm{A} 549,1.11 \mathrm{E}+07 \pm 2.21 \mathrm{E}+06)$ at higher E:T ratios (online supplemental figure S2D, figure 3A). Furthermore, the results of CAR-specific lysis assay also supported the higher cytotoxic function of 21D4 CAR-T cells $(10: 1,37.9 \% \pm 5.0 \%)$ against CD19 mutated tumor cells than that of FMC63 CAR-T cells $(10: 1,19.3 \% \pm 5.8 \%)$. (figure 3B), suggesting that there is a difference between the cytotoxic function of FMC63 and 21D4 CAR-T cells. We next investigated which exon of CD19 is most important for the recognition of CAR-T cells. For this, CD19 exon 1, 2, 3, or 4 was depleted and cloned into pCDH-EF1-IRESPuro vector. Finally, we obtained four different H322 cell lines with deleted CD19 exon (online supplemental figure S3A, B). Then these cells were cocultured with CAR-T cells at E:T ratio of 10:1. Our results showed that FMC63 CAR-T cells were unable to kill the target cells with 1, 2, or 3 exon deleted, but 21D4 CAR-T cells could still exert an effector specific function against these cells (figure 3C,D). However, neither FMC63 nor 21D4 CAR-T cells could eradicate CD19 positive H322 cells, in which the CD19 exon four was deleted (figure 3C,D). This analysis revealed that the deletion of CD19 exons 1-3 or mutation in exon 3 are sufficient for 21D4 CART-19 cells to exert their cytotoxic function.

\section{D4 CAR-T cells exhibit enhanced potency against mutated CD19 cells in vivo}

Based on the observation that 21D4 CAR-T cells exhibit higher potency against mutated $\mathrm{CD} 19^{+}$cells in vitro, we speculated whether CAR-T cells with different scFVs fragments could exert similar outcomes in vivo. $5 \times 10^{6}$ Raji/Luc cells were injected through tail vein and simultaneously $1 \times 10^{6}$ wild type or mutated CD19+-H322/Luc cells were inoculated subcutaneously into the immune-deficient mice 1 week before CAR-T treatment, respectively. Tumor burdens across the different groups were balanced by BLI analyzes before CAR-T infusion. Thereafter, $1 \times 10^{7}$ CAR-T 

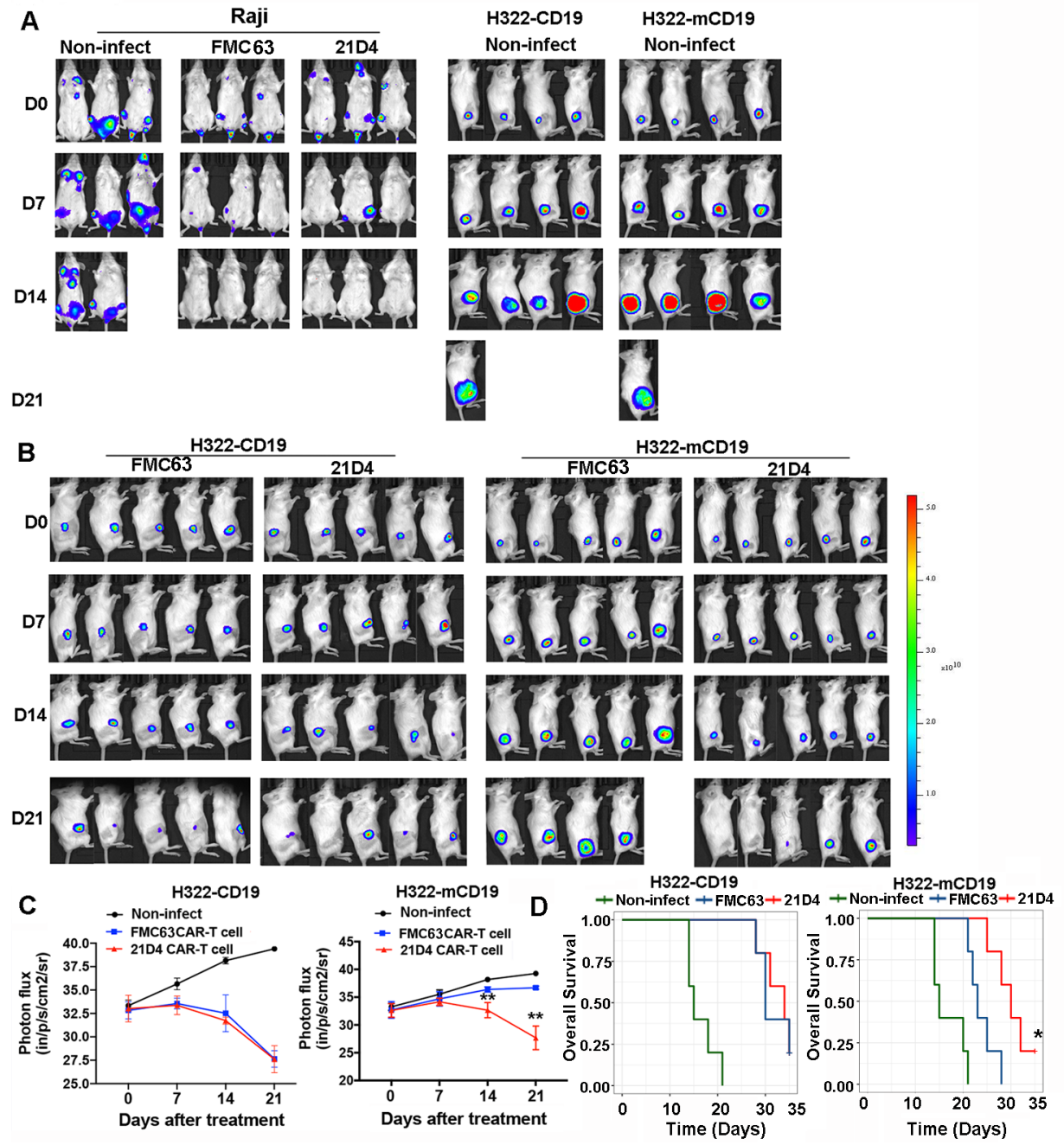

Figure 4 Antitumor ability of CD19 CAR-T cells in vivo. (A-C) The efficacy of CD19 CAR-T cells was examined using a mouse model. Briefly, $1 \times 10^{6}$ luciferase-transgenic Raji cells were inoculated into SCID-beige mice via tail on day 7 . The $1 \times 10^{6}$ wild type or mutated CD19 positive H322/Luc cells were subcutaneously injected into the mice. On day $0,1 \times 10^{7}$ non-infected T cells or FMC63 and 21D4 CAR-T cells were injected intravenously (intravenous). Then, tumor growth was monitored via bioluminescent imaging test at indicated time points. The tumor growth was controlled by both CAR-T cells in wild type CD19 model. Moreover, 21D4 CAR-T cells showed better antitumor function than that of FMC CAR-T cells in mutated CD19 model. The expression values of the photon flux were transformed into log2 values for further analysis. (D) The survival curves were analyzed using the log-rank test, and the statistical result showed that a significant difference between FMC63 and 21D4 CAR-T cells treated H322-mCD19 mice. ${ }^{*} p<0.05,{ }^{\star *} p<0.01$. CAR, chimeric antigen receptor.

cells cultured for 10 days were infused to mice via tail, and mock T cells were used as control. Both FMC63 and 21D4 CAR-T cells demonstrated anti-lymphoma activity in all the treated mice on BLI examination compared with that in uninfected $\mathrm{T}$ cell-treated mice (figure $4 \mathrm{~A}$ ). Moreover, FMC63 and 21D4 CAR-T cells effectively controlled CD19 ${ }^{+}$H322 tumor cell growth, while the tumor growth was observed in mice treated with control $\mathrm{T}$ cells (figure $4 \mathrm{~B}$ ). We also assessed the antitumor effect of FMC63 and 21D4 CAR-T cells in mutated CD19 ${ }^{+}$ H322 model. As shown in figure 4B,C, 21D4 CAR-T cells significantly controlled mutated $\mathrm{CD} 19^{+}$tumor growth when compared with FMC63 CAR-T cells. Meanwhile, the 21D4 CAR-T cells treated H322-mCD19 mice had a longer overall survival than the mice treated with FMC63 CAR-T cells (figure 4D). These results suggest that there is a difference in effector-specific function of CD19 CAR comprising scFV antibody fragments FMC63 or 21D4.

\section{Antitumor ability of 21D4 CAR-T cells in CD19 mutated patients with $B$ cell lymphoma}

Whether this type of mutation appears in patients with NR to CAR-T cell therapy is not clear. Therefore, we further analyzed the mutations in $\mathrm{CD} 19^{+}$cells from two different patients with NR and one patient with CR as a control. The clinical characteristics of these patients are presented in table 1 . All of the patients exhibited progression after receiving chemotherapy regimen. Patient 2 was treated according to the anti-CD19 CAR protocol and achieved a CR state in 24 months. Two patients with lymphoma resisted the CAR-T cell therapy and died due to disease progression. We detected CAR-T cells by qRT-PCR analysis 
Table 1 Clinical characteristics of patients with B cell malignancy

\begin{tabular}{lllllllll}
\hline $\begin{array}{l}\text { Patient } \\
\text { no. }\end{array}$ & Malignancy & $\begin{array}{l}\text { Age } \\
\text { (years) }\end{array}$ & Sex & Chemotherapy regimens & $\begin{array}{l}\text { 年 } \\
\text { involvement }\end{array}$ & $\begin{array}{l}\text { Total. of } \\
\text { CAR-T } \\
\text { cells } \\
\text { infused } \\
\left(\times 10^{6}\right)\end{array}$ & Response & Mutation \\
\hline 1 & HGBCL & 24 & Female & $\begin{array}{l}\text { R-CHOP+Hyper CVAD A and } \\
\text { B }\end{array}$ & Yes & 21.8 & Relapse & p.163. R-L \\
2 & MCL & 54 & Male & CHOP+DHAP & Yes & 32.2 & CR & No \\
3 & DLBLC & 54 & Male & R-CHOP+R-ECHOP & Yes ${ }^{*}$ & 105 & NR & p.174. L-V \\
4 & DLBLC & 47 & Female & R-CHOP & No & 35.5 & NR & p.174. L-V \\
\hline
\end{tabular}

${ }^{*}$ The bone marrow involvement was occurred on day 37 after CAR-T cells infusion.

CAR, chimeric antigen receptor; DLBLC, diffuse large B cell lymphoma; HGBCL, high grade B cell lymphoma; MCL, mantle cell lymphoma.

in the peripheral blood samples of these patients obtained after infusion and observed an increase in the number of CAR-T cells in these patients (figure 5A). Patient 3 with NR exhibited bone marrow involvement on day 37 and died on day 42 after infusion of CAR-T cells. Patient 4 with NR also died on day 87 after treatment with CAR-T cells. We further found that the percentage of malignant $\mathrm{CD} 19^{+} \mathrm{B}$ cells in bone marrow or tumor tissues from the two NR patients after CAR-T cell treatment were $85.1 \%$ and $99.8 \%$, respectively (data not shown). Additionally, these two patients possessed a point mutation in exon 3 of $C D 19$ (p.174 L >V, figure 5B). However, the patient with CR had no such mutation. Further analysis revealed that 21D4 CAR-T cells exhibit better effector-specific function compared with FMC63 CAR-T cells when co-cultured with $\mathrm{CD} 19^{+}$cells from patients with NR (figure 5C). Altogether, the patients possessing point mutation in $\mathrm{CD}_{1} 9^{+}$ cells (exon 3, p.174 L $>$ V) might exhibit resistance to CD19 CAR-T cell therapy, and these mutated cells can be effectively eradicated by 21D4 CAR-T cells.

\section{DISCUSSION}

CD19-based immunotherapy has changed the pattern of treatment of relapsed or refractory B cell lymphomas. ${ }^{20-22}$ Patients with a previously unsatisfactory outcome have now been reported to achieve CR and long-term disease remission. ${ }^{23}$ However, clinical data have revealed that a subset
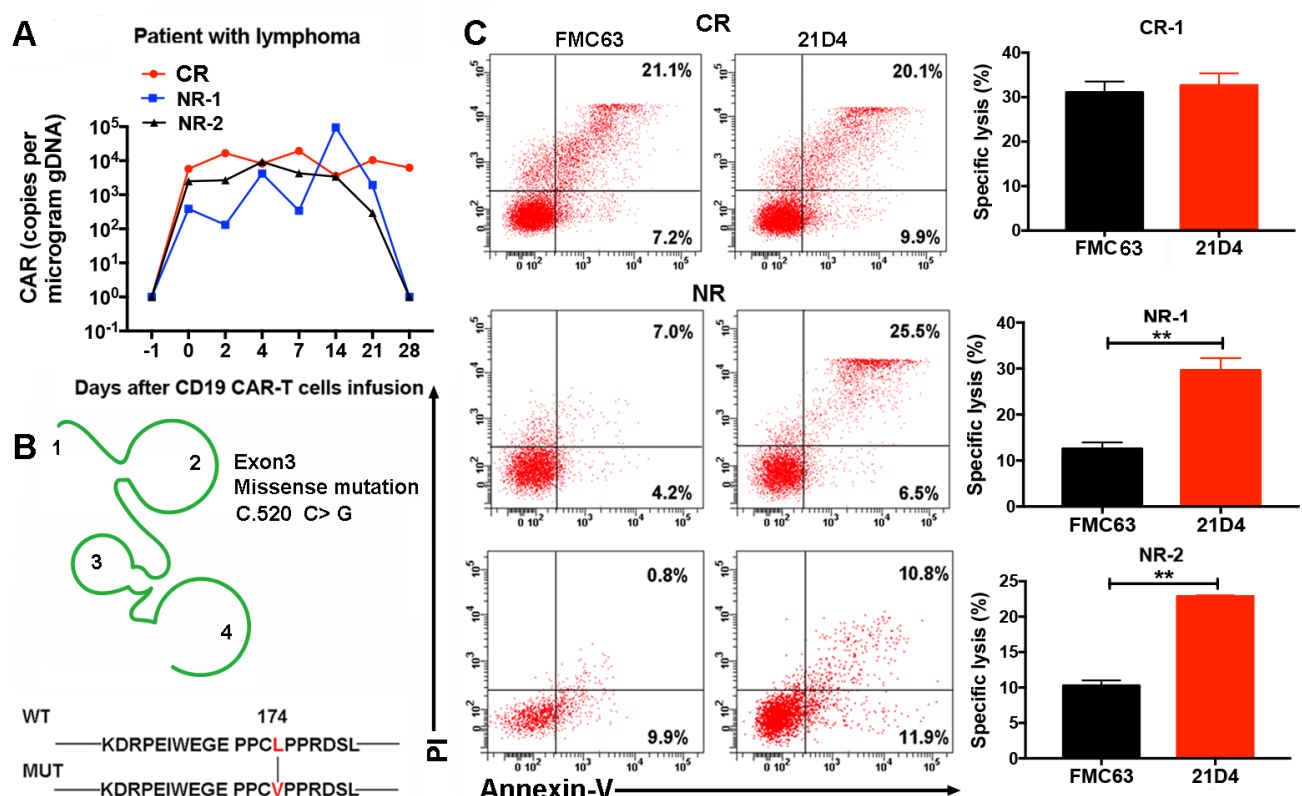

Figure 5 21D4 CAR-T cells exhibit a potent cytotoxicity against mutated CD19+ cells derived from patients with no response (NR). (A) Quantitative real-time-PCR analysis of CAR-T cells in peripheral blood. (B) Lymphoma patients with NR had a point mutation in exon 3 (P. $174 \mathrm{~L}>\mathrm{V} / \mathrm{C} .520 \mathrm{C}>\mathrm{G}$ ) of CD19. (C) FMC63 or 21D4 CAR-T cells were cocultured with mutated CD19 ${ }^{+} \mathrm{B}$ cells derived from patients at E:T ratio of $5: 1$ for 6 hours. Lysis of $C D 19^{+}$cells was detected and 21D4 CAR-T cells exhibited higher lysis ability than FMC63 CAR-T cells in the patients with NR. ${ }^{* *} \mathrm{p}<0.01$. CAR, chimeric antigen receptor; CR, complete response. 
of lymphoma or B-ALL patients that achieves remission subsequently undergoes relapse. Our clinical trial with FMC63-CD19 CAR supports the fact that some patients exhibited relapse and resistance after the treatment. Our results showed that infusing cells against lymphoma is effective and safe. One patient with HGBCL received autologous CD19 CAR-T cell therapy. We observed that the number of CAR-T cells in the blood rapidly increased on day 14 and decreased after eliminating the tumor cells on day 28 as detected by flow cytometry. Meanwhile, the $\mathrm{CD} 19^{+}$cells were found to be significantly decreased on day 14 and disappeared by day 28 after CAR-T cell infusion. This finding is similar with the mechanism of $\mathrm{T}$ cell activation because higher CAR-T cell activity against target cells may enhance the responses of effector T cells and stimulate CAR-T cell proliferation. It is noticeable that CAR-T cells in this patient were durable and persisted in vivo, but the left breast tumor tissue was found to express $84.1 \%$ CD19 protein on day 180 , indicating a CD19 ${ }^{+}$ recurrence of this patient. Moreover, this $\mathrm{CD}_{1} 9^{+}$relapse was also verified by immunohistochemical analysis in the department of pathology (data not shown). The number of CAR-T cells was slightly increased at this stage of recurrence, and then decreased to $0.6 \%$ (at day 579) after the tumor was removed by surgery in combination with anti-PD-1 therapy. However, CAR-T cells increased in number in the first 14 days and rapidly disappeared in the two patients with NR, suggesting that these patients initially resisted the treatment.

Recent studies have reported that the reasons of relapse includes CAR-T cell persistence and loss or downregulation of the targeted CD19 antigens. ${ }^{23}$ Elena et al have reported the underlying mechanism of resistance to CD19 CAR-T cells and suggested that exon 2 of CD19 is frequently spliced out, leading to the inhibition of CD19 protein expression. Moreover, the acquired genetic mutations in CD19 exons 2-5 are the major mechanisms of CD19 loss. Ramakrishna et al also found that low target antigen expression negatively impacts the in vitro and in vivo CAR-T cell functionality and impairs in vivo persistence of CAR-T cells. ${ }^{24}$ To identify the reason behind the immune escape from CAR-T cells in the patient exhibiting $\mathrm{CD}_{1} 9^{+}$relapse, we first compared the expression of transcription factors, inhibitory markers, and genes associated with cytotoxicity in CAR-T cells before and after infusion. Our results indicated that functional CAR-T cells persisted in blood until recurrence. We further analyzed the molecular characteristics and mutations of B cells. The relapsed $\mathrm{CD}_{1} 9^{+}$cells expressed transcription factors at high levels. For example, the expression IRF4, a member of the interferon regulatory factor family, which is associated with the progression of lymphoma, was increased. ${ }^{25} M E F 2 B$, the most divergent and least studied protein of the MEF2 family, has a unique role in non-Hodgkin lymphomas. ${ }^{26}$ The combination of $M Y C$ and/or BCL2 with BCL6 in lymphoma has been linked to unfavorable clinical outcomes. ${ }^{27}$ The expression level of other genes such as CD79B, MYD88 and RELA were also high on day 180 , which may be associated with the development of lymphoma. ${ }^{28}{ }^{29}$ Next-generation sequencing of DNA from $\mathrm{CD} 19^{+}$cells in relapsed tumor tissues revealed that this patient had a mutation on exon 3 of $C D 19$ (p.163.
$\mathrm{R}-\mathrm{L})$, but it was not detected in the $\mathrm{CD} 19^{+}$cells from bone marrow prior to CAR-T cell therapy. The other patients with CR (4/14) had no relapse after autologous CD19 CAR-T cell therapy in our clinical trial, ${ }^{30}$ and one of these patients exhibited no mutation in $\mathrm{CD}_{1} 9^{+}$cells before treatment. For the patients with $\mathrm{CD} 19^{-}$relapse, various mutations were detected in $C D 19$ isolated from bone marrow DNA. However, these mutations were not detectable before CAR-T cell treatment. Orlando et al sequenced samples collected at various time points during treatment, and they demonstrated that the genetic variants at relapse were not detectable in the specimens before relapse. ${ }^{11}$ This treatment failure was due to growth of clones with CD19 mutations, which led to a decrease in the expression of CD19 protein on the surface of cells that were under selective pressure from CAR-T cells. ${ }^{31}$ Moreover, each relapsed patient with ALL has different mutations, and $4 / 5$ patients had the mutation on exon 3 . The data suggest that patients who received CAR-T cell therapy might be prone to mutation on exon 3. The mutation identified in $\mathrm{CD} 19^{+}$relapse patient in our study was also different from any other $\mathrm{CD} 19^{-}$relapse mutation that reported before, but it also occurred in exon $3 .^{11}$ The two patients with NR acquired the mutation in $C D 19$ (p.174 (exon3) $\mathrm{L}>\mathrm{V}$ ) on 1 month after infusion with CD19 CAR-T cells. It is a pity for that the samples can't be obtained before and after treatment to perform WES. Whether the selective pressure from CAR-T cells can lead to outgrowth of clones with this CD19 mutations in NR patients or not is still unclear. We further found that the FMC63 CAR-T cells could kill the wild type CD19 ${ }^{+}$ cells, but not of the mutated $\mathrm{CD} 19^{+}$cells derived from tumor tissues or bone marrow. These data indicate that CD19 mutation may contribute to the immune escape.

Interestingly, most of patients with ALL continued to generate $\mathrm{CD} 22^{+}$cells at the time of relapse. Therefore, the application of CD22 CAR-T cells could be a promising treatment strategy. ${ }^{32-34}$ Alternatively, CAR engineering could be used to enhance the affinity of the scFv activity against tumor cells with low antigen density. It indicates that by altering the affinity of EGFR and/or HER2 CARs, it is possible to recognize as low levels of antigens as expressed on a normal tissue. However, a research group demonstrated that CD22 CAR-T cells with high affinity become terminally differentiated when stimulated by CD22 ${ }^{\text {low }}$ leukemia cells and exhibited no improvement in function. ${ }^{24}$ There are limited strategies that can be implemented for the treatment of patients with $\mathrm{CD} 19^{+}$ mutations. In our study, we compared the effector-specific function of FMC63 and 21D4 CAR-T cells against the mutated CD19 tumor cells. Our results showed that 21D4 CAR-T cells had a potent cytotoxic effect against the mutated $\mathrm{CD} 19^{+}$cells (p.163 R $>$ L) both in vitro and in vivo. Further analysis revealed that 21D4 CAR-T cells also can eradicate CD19 ${ }^{+}$cells with p.174 (exon3) L $>$ V mutation. Previous reports have shown that the epitope recognized by FMC63 containing the residues, which are distant at sequence but proximal at space. ${ }^{35}$ Hence, the epitopes of CD19 are complicated in terms of antibody recognition. Moreover, the affinity of antibody may play a key role in impacting the CAR-T cell response. Recently, the epitope of 21D4 still remains unknown. To compare the 
functional differences between FMC63 and 21D4 CAR-T cells, we constructed $\mathrm{CD} 19^{+}$cells with deleted exon 1, 2, 3, or 4. HIB19 can be completely blocked the binding of FMC63 to cells by competitive binding experiments, suggesting that FMC63 and HIB19 may bind to the same or overlapping epitopes on CD19. ${ }^{12} 36$ We found that in the exon deleted cells, the expression of CD19 was not changed at transcriptional level, but was significantly decreased at protein level (testing by HIB19, data not shown). Our data also revealed that 21D4 CAR-T cells possess high antitumor capacity for the eradication of $\mathrm{CD} 19^{+}$cells with exon 1 , 2, or 3 deletion, while FMC63 CAR-T cells could not exert the same effect on the target cells. Our results suggest that these two kinds of CAR-T cells may possess different binding sites for CD19. Based on these findings, we suggest that 21D4 CAR-T cells may be applied as an individualized treatment strategy for some recurrent patients with point mutation in $\mathrm{CD} 19^{+}$cells.

In summary, FMC63 CAR-T cells showed limited cytotoxic efficacy in patients with CD19 mutations, leading to relapse after the CAR-T cell therapy. Interestingly, 21D4 CAR-T cells could exert cytotoxic effect on B cells with CD19 mutations both in vitro and in vivo, which may thus provide an alternative strategy for establishing a treatment for lymphoma patients with CD19 mutations.

Acknowledgements We thank the patients and healthy donors for their signed informed consent forms in this experiment.

Contributors YZ: designed research; ZZ, YT, FL, and CY: performed experiments, data collection, and statistical analyses; $\mathrm{XC}$ and $\mathrm{XZ}$ : enrolled patients; $\mathrm{YZ}$ and $\mathrm{JL}$ : revised the manuscript; and all authors were approved the final version.

Funding This study was supported by the National Key Research and Development Program of China (No. 2018YFC1313400) and the National Natural Science Foundation of China (Grant No. U1804281, 81771781, and 81702810).

Competing interests None declared.

Patient consent for publication Not required.

Ethics approval All the volunteer and patient were recruited, following the approval by the Ethics Committee Board of the First Affiliated Hospital of Zhengzhou University. Animal studies were approved by the Animal Care and Ethics Committee of the First Affiliated Hospital of Zhengzhou University.

Provenance and peer review Not commissioned; externally peer reviewed.

Data availability statement № data are available.

Open access This is an open access article distributed in accordance with the Creative Commons Attribution Non Commercial (CC BY-NC 4.0) license, which permits others to distribute, remix, adapt, build upon this work non-commercially, and license their derivative works on different terms, provided the original work is properly cited, appropriate credit is given, any changes made indicated, and the use is non-commercial. See http://creativecommons.org/licenses/by-nc/4.0/.

\section{ORCID iDs}

Feng Li http://orcid.org/0000-0002-3895-0437

Yi Zhang http://orcid.org/0000-0001-9861-4681

\section{REFERENCES}

1 June $\mathrm{CH}$, O'Connor RS, Kawalekar OU, et al. CAR T cell immunotherapy for human cancer. Science 2018;359:1361-5.

2 Maude SL, Laetsch TW, Buechner J, et al. Tisagenlecleucel in children and young adults with B-cell lymphoblastic leukemia. N Engl J Med 2018;378:439-48.

3 Ruella M, Maus MV. Catch me if you can: leukemia escape after CD19-Directed T cell immunotherapies. Comput Struct Biotechnol J 2016;14:357-62.
4 Ruella M, Barrett DM, Kenderian SS, et al. Dual CD19 and CD123 targeting prevents antigen-loss relapses after CD19-directed immunotherapies. J Clin Invest 2016;126:3814-26.

5 Qin $\mathrm{H}$, Dong Z, Wang X, et al. CAR T cells targeting BAFF-R can overcome CD19 antigen loss in B cell malignancies. Sci Transl Med 2019;11:eaaw9414.

6 Sotillo E, Barrett DM, Black KL, et al. Convergence of acquired mutations and alternative splicing of CD19 enables resistance to CART-19 immunotherapy. Cancer Discov 2015;5:1282-95.

7 Gardner R, Wu D, Cherian S, et al. Acquisition of a CD19-negative myeloid phenotype allows immune escape of MLL-rearranged B-ALL from CD19 CAR-T-cell therapy. Blood 2016;127:2406-10.

8 Grupp SA, Kalos M, Barrett D, et al. Chimeric antigen receptormodified T cells for acute lymphoid leukemia. N Engl J Med 2013;368:1509-18.

9 Zhang Z, Liu S, Zhang B, et al. T cell dysfunction and exhaustion in cancer. Front Cell Dev Biol 2020;8:17.

10 Kasakovski D, Xu L, Li Y. T cell senescence and CAR-T cell exhaustion in hematological malignancies. J Hematol Oncol 2018;11:91

11 Orlando EJ, Han X, Tribouley C, et al. Genetic mechanisms of target antigen loss in CAR19 therapy of acute lymphoblastic leukemia. Nat Med 2018;24:1504-6.

12 Ruella M, Xu J, Barrett DM, et al. Induction of resistance to chimeric antigen receptor $\mathrm{T}$ cell therapy by transduction of a single leukemic $\mathrm{B}$ cell. Nat Med 2018;24:1499-503.

13 Liu Y, Chen X, Wang D, et al. Hemofiltration successfully eliminates severe cytokine release syndrome following CD19 CAR-T-Cell therapy. J Immunother 2018;41:406-10.

14 Mueller KT, Maude SL, Porter DL, et al. Cellular kinetics of CTL019 in relapsed/refractory $B$-cell acute lymphoblastic leukemia and chronic lymphocytic leukemia. Blood 2017;130:2317-25.

15 Maude SL, Frey N, Shaw PA, et al. Chimeric antigen receptor T cells for sustained remissions in leukemia. N Engl J Med 2014;371:1507-17.

16 de Miranda NFCC, Georgiou K, Chen L, et al. Exome sequencing reveals novel mutation targets in diffuse large $B$-cell lymphomas derived from Chinese patients. Blood 2014;124:2544-53.

17 de Miranda NFCC, Peng R, Georgiou K, et al. DNA repair genes are selectively mutated in diffuse large B cell lymphomas. J Exp Med 2013;210:1729-42

18 Guan T, Dominguez CX, Amezquita RA, et al. ZEB1, ZEB2, and the miR-200 family form a counterregulatory network to regulate $C D 8^{+} \mathrm{T}$ cell fates. J Exp Med 2018;215:1153-68.

19 Xia Y, Xu-Monette ZY, Tzankov A, et al. Loss of Prdm1/Blimp-1 function contributes to poor prognosis of activated B-cell-like diffuse large B-cell lymphoma. Leukemia 2017;31:625-36.

20 Schuster SJ, Svoboda J, Chong EA, et al. Chimeric antigen receptor $\mathrm{T}$ cells in refractory $\mathrm{B}$-cell lymphomas. $\mathrm{N}$ Engl J Med 2017;377:2545-54.

21 Kochenderfer JN, Dudley ME, Kassim SH, et al. Chemotherapyrefractory diffuse large $\mathrm{B}$-cell lymphoma and indolent B-cell malignancies can be effectively treated with autologous $T$ cells expressing an anti-CD19 chimeric antigen receptor. J Clin Oncol 2015;33:540-9.

22 Brudno JN, Kochenderfer JN. Chimeric antigen receptor T-cell therapies for lymphoma. Nat Rev Clin Oncol 2018;15:31-46.

23 Park JH, Rivière I, Gonen M, et al. Long-term follow-up of CD19 CAR therapy in acute lymphoblastic leukemia. N Engl J Med 2018;378:449-59.

24 Ramakrishna S, Highfill SL, Walsh Z, et al. Modulation of target antigen density improves CAR T-cell functionality and persistence. Clin Cancer Res 2019;25:5329-41.

25 Gualco G, Weiss LM, Bacchi CE. MUM1/IRF4: a review. App/ Immunohistochem Mol Morphol 2010;18:301-10.

26 Pon JR, Marra MA. Mef2 transcription factors: developmental regulators and emerging cancer genes. Oncotarget 2016;7:2297-312.

27 Rosenthal A, Younes A. High grade B-cell lymphoma with rearrangements of myc and $\mathrm{Bcl} 2$ and/or BCL6: double hit and triple hit lymphomas and double expressing lymphoma. Blood Rev 2017;31:37-42

28 Onaindia A, Medeiros LJ, Patel KP. Clinical utility of recently identified diagnostic, prognostic, and predictive molecular biomarkers in mature B-cell neoplasms. Mod Pathol 2017;30:1338-66.

29 Gasparini C, Celeghini C, Monasta L, et al. NF- $\kappa B$ pathways in hematological malignancies. Cell Mol Life Sci 2014;71:2083-102. 
30 Chen X, Li X, Liu Y, et al. A phase I clinical trial of chimeric antigen receptor-modified $\mathrm{T}$ cells in patients with relapsed and refractory lymphoma. Immunotherapy 2020;12:681-96.

31 Ghorashian S, Kramer AM, Onuoha S, et al. Enhanced CAR T cell expansion and prolonged persistence in pediatric patients with all treated with a low-affinity CD19 CAR. Nat Med 2019;25:1408-14.

32 Jia H, Wang Z, Wang Y, et al. Haploidentical CD19/CD22 bispecific CAR-T cells induced MRD-negative remission in a patient with relapsed and refractory adult B-ALL after haploidentical hematopoietic stem cell transplantation. $J$ Hematol Oncol 2019;12:57.
33 Qin H, Ramakrishna S, Nguyen S, et al. Preclinical development of bivalent chimeric antigen receptors targeting both CD19 and CD22. Mol Ther Oncolytics 2018;11:127-37.

34 Wei J, Han X, Bo J, et al. Target selection for CAR-T therapy. J Hematol Oncol 2019;12:62.

35 Klesmith JR, Wu L, Lobb RR, et al. Fine epitope mapping of the CD19 extracellular domain promotes design. Biochemistry 2019;58:4869-81.

36 Du X, Beers R, Fitzgerald DJ, et al. Differential cellular internalization of anti-CD19 and-CD22 immunotoxins results in different cytotoxic activity. Cancer Res 2008;68:6300-5. 